\title{
L'environnement pousse à un renouveau méthodologique et analytique
}

\author{
Entretien avec Jean Foyer
}

Jean Foyer et Arthur Laurent

Jean Foyer est sociologue à l'ISCC (CNRS/Paris IV/UPMC). Ses recherches ont porté sur des objets comme les mouvements sociaux indigènes et environnementaux, sur les controverses autour de la bioprospection et des OGM ou sur les politiques publiques de conservation de l'agro-biodiversité au Mexique. Il poursuit actuellement ses recherches sur la gouvernance mondiale de l'environnement. Il a notamment coordonné le projet Ecoverio qui a proposé une ethnographie collective du Sommet de la terre Rio+20 et co-coordonne actuellement le projet Climacop sur la COP 21. Il a notamment publié Il était une fois la bio-révolution (2010), et coordonné les publications Regards croisés sur Rio+20. La modernisation écologique à l'épreuve (CNRS Éditions, 2015) et Globalising the Climate: COP21 and the climatisation of global debates (Routledge, 2017), avec Stefan Aykut et Edouard Morena.

Vos recherches ont porté sur des enjeux environnementaux qui dégagent clairement des dimensions transnationales. Pouvez-vous nous expliquer comment cette dimension vous est apparue et comment l'avez-vous traitée?

Un de mes premiers thèmes d'études a étél'analyse d'un conflit socio-environnemental dans une réserve de la biosphère du Sud du Mexique, au Chiapas. Il s'agissait entre autres de voir comment un cadre environnemental pensé depuis une institution internationale comme l'UNESCO venait se superposer à d'autres formes d'appropriation du territoire et entrait en tension avec elles. Il s'agissait aussi de voir comment la logique environnementale d'acteurs conservationnistes étrangers entrait en conflit avec celles plus politiques et agraristes d'acteurs locaux, en l'occurrence, les mouvements paysans et indigènes zapatistes. Tout ça dans un contexte local où affluait également toute une foule d'ONG autour du mouvement zapatiste et où l'État mexicain restait très présent pour des questions anti-insurrectionnelles. Ce premier terrain était donc très formateur pour comprendre les imbrications des logiques locales, nationales et transnationales, même si à chaque fois, on peut questionner la pertinence de ces catégories. Même si mon entrée était plus locale, disons donc que la dimension transnationale était déjà très présente.

Même chose dans les controverses autour des biotechnologies, toujours depuis le Mexique, que j’ai étudiées ensuite. Au niveau territorial comme au niveau des acteurs, 
les controverses sur la bioprospection ou les OGM impliquaient des universités américaines ou européennes, des firmes et des ONG transnationales, des médias étrangers et débordaient donc largement du cadre mexicain. Par exemple, je me suis intéressé à ce que l'on a appelé les experts-militants de la biodiversité qui en combinant une expertise de long terme avec des registres d'action empruntés au militantisme, notamment en ligne, sont d'une efficacité remarquable pour faire circuler au niveau mondial des cadrages sur des questions comme les OGM, la biopiraterie, les nanotechnologies ou la biologie de synthèse. Des ONG connues comme Greenpeace peuvent entrer dans cette catégorie, mais celles qui y correspondent le mieux sont généralement moins visibles et connues : je pense à l'ETC Group basé au Canada ou au Third World Network en Asie par exemple.

Comme beaucoup de collègues donc, notamment dans le courant anglo-saxon de la Political Ecology, je suis remonté peu à peu vers ce niveau transnational pour m'intéresser à la fabrique de cette gouvernance globale de l'environnement, notamment lors de méga-événements transnationaux. C'est comme ça que l'on a décidé d'étudier avec des collègues le Sommet de la Terre de Rio+20 en 2012 et plus récemment, la COP21 à Paris. Sur la base d'une méthodologie collaborative adaptée à ce type d'objet devant lequel un chercheur individuel est souvent perdu, à Rio+20, on observait «en temps réel » l'émergence d'un cadrage comme celui de l'économie verte dont la mise à l'agenda a été un relatif échec d'ailleurs. On regardait plus généralement comment cet événement constituait une mise à l'épreuve pour l'approche globale en termes de modernisation écologique, c'est-à-dire une version très gestionnaire et technocratique de l'écologie qui cherche à rendre compatible les préoccupations environnementales avec celle du marché notamment. En ce qui concerne la COP21, nous l'avons analysée comme un espace-temps d'intense "climatisation" du monde, un moment où les grands enjeux mondiaux des questions environnementales (biodiversité, océan, forêt) aux questions de commerce, en passant par celles d'énergie, de développement, de sécurité ou même de religion, étaient repensés en termes climatiques. Une nouvelle fois, notre intérêt comme notre méthode ne relève pas vraiment des Relations Internationales, même si c'est connexe. Ce qui nous intéresse, c'est montrer comment se construisent ce globalisme environnemental et sa gouvernance pour être ensuite capables de reconstituer les chaînes de traduction entre les différents niveaux local, national, global.

En particulier, selon vos recherches les enjeux environnementaux modifient-ils l'exercice du pouvoir?

Les enjeux environnementaux modifient la politique au sens où ils élargissent le spectre de la Polis en y faisant entrer des animaux, des plantes, des paysages, le climat et toute une série d'autres non-humains dont on ne peut plus ne pas tenir compte. Les débats actuels sur la pertinence d'accorder des droits à certaines entités naturelles sont éloquents à ce propos. En ce sens, les enjeux environnementaux ont plus recomposé le monde à gouverner que les manières d'exercer le pouvoir à proprement parler. Sur ce point quand même, on peut peut-être penser que l'environnement, du fait de l'in- 
fluence de l'écologie scientifique qui en structure souvent les cadrages, mais plus généralement du fait de l'élargissement du spectre des éléments à gouverner qu'elle suppose, tend vers des formes plus systémiques et moins centralisées d'exercice du pouvoir. Ce n'est sans doute pas un hasard si l'essentiel des partis Verts européens sont très fédéralistes. La montée des enjeux environnementaux est concomitante des transformations plus générales d'exercice du pouvoir vers ce qu'on appelle la gouvernance et il est difficile de savoir lequel de ces deux mouvements a influencé l'autre, même s'il y a très certainement eu des effets réciproques.

Les enjeux environnementaux tendraient ainsi à favoriser des formes de gestion multi-partenariales ou transversales plus que des décisions centralisées, mais ceci ne doit pas faire oublier qu'il reste des rapports de force sectoriels très forts dans les politiques internationales ou nationales. Aussi transversales soient-elles, les questions environnementales n'ont par exemple pas modifié structurellement les agendas politiques ni l'allocation des moyens pour les mettre en œuvre. Je connais très peu de pays où le ministère de l'Environnement a plus de moyens et d'influence que celui de l'agriculture ou à plus forte raison encore, celui des finances. La France a été élue championne environnementale pour son rôle dans la COP21, mais elle recule sur la taxe carbone et fait construire un nouvel aéroport au nom d'intérêts économiques et des intérêts de l'État.

La notion de gouvernance a notamment été appliquée aux enjeux environnementaux, vous semble-t-elle effectivement opératoire?

Le problème avec le terme de gouvernance, c'est que c'est à la fois un concept de sciences politiques et une sorte de projet politique, avec une dimension normative forte et je pense qu'il faut bien faire la distinction entre les deux. Comme concept, je pense qu'il est utile par rapport à celui de gouvernement notamment pour penser ces formes de politiques moins centrées sur l'État, multi-partenariales (qui incluent le secteur privé, les ONG, les médias, etc.) et multi-niveaux. Pour décrire les enjeux environnementaux qui correspondent souvent à ce type de dynamique, je pense que ce terme est donc très utile. Certains collègues critiques refusent de l'utiliser au prétexte que l'utiliser n'est pas neutre au sens où ça servirait le projet politique de la gouvernance. Ce terme s'est en effet diffusé dans un contexte idéologique particulier, post-guerre froide, et véhicule plus ou moins implicitement un imaginaire managérial et technocratique où le monde est par nature gouvernable si on trouve le meilleur niveau (le principe de subsidiarité) et les meilleurs compromis entre acteurs. Évidemment, c'est oublier tout d'abord qu'il existe des situations de gouvernabilité très précaires du fait de conflits ou de la faible structuration des parties prenantes par exemple. La gouvernabilité d'une forêt en Suède n'est pas celle d'une forêt en RDC pour toutes les raisons que vous pouvez imaginer. Ensuite, les différents acteurs ne négocient pas sur un pied d'égalité. Dans un projet extractiviste, une multinationale pétrolière et une organisation communautaire négocieront difficilement selon les mêmes conditions. Tant qu'on n'oublie 
pas d'évoquer le contexte et les questions de rapport de force, je pense donc que le concept de gouvernance permet de combler un vide dans les instruments des sciences sociales sur l'environnement et plus généralement.

Quelles sont selon vous les grandes caractéristiques des enjeux environnementaux au niveau global actuellement?

Il ne faut pas oublier en premier lieu qu'il n'y a que peu de questions environnementales qui arrivent à se constituer comme enjeu global. Devenir un enjeu environnemental global nécessite tout un travail scientifique, militant, institutionnel pour être reconnu comme enjeu global. On ne doit pas naturaliser ce globalisme, d'où la nécessité d'en étudier la fabrique. Actuellement, même si on peut penser que plus de questions environnementales se sont constituées en enjeu global, seuls trois régimes internationaux, celui du climat, celui de la biodiversité et celui de la désertification, fonctionnent effectivement, et encore, avec des disparités énormes. La convention sur la désertification est moribonde et celle sur la biodiversité tend à copier le régime climatique qui a une force d'attraction sur tous les autres enjeux environnementaux. Si on prend les neuf limites planétaires évoquées dans le célèbre article de Rockstrom, on se rend compte que d'une certaine manière, la climatisation des enjeux environnementaux invisibilise d'autres problèmes également très importants comme les cycles de l'azote et du phosphore, les pollutions chimiques ou l'acidification des océans. Donc, une des premières caractéristiques de ces enjeux est qu'au moins politiquement, leur globalisme doit être construit et que ces enjeux constituent un champ au sens où, d'une certaine manière, ils sont en concurrence.

L'autre élément qui me paraît important, non pas tant sur ces enjeux eux-mêmes que sur leur gouvernance à ce niveau global, est ce que James Carrier et Paige West (ont appelé leur « virtualisme ». La gouvernance des enjeux globaux est en effet dans une large mesure virtuelle au sens où elle ne se traduit que très rarement dans les faits par des réalisations qui correspondent à ce qui est édicté au niveau international/global. Une vision un peu superficielle de la gouvernance globale veut que du fait de son échelle «supérieure » par rapport au national et au local, on pense qu'elle s'impose mécaniquement à ces niveaux. Penser ça, c'est oublier toutes les chaînes de traduction de ces politiques avant qu'elles n'atterrissent au niveau local, avec tous les effets de distorsion, contournement et réappropriation auxquels ces traductions peuvent donner lieu. Si on prend les modèles de conservation des Aires naturelles protégées, à la fin des années 70, au niveau de l'UNESCO on était déjà sur un modèle participatif censé inclure les populations locales. Mais au niveau local, on était le plus souvent encore à l'époque des "parcs de papiers", des réserves naturelles qui n'avaient une existence qu'au niveau législatif. On est aujourd'hui dans un paradigme connexionniste avec les fameux corridors de biodiversité, mais, avant de penser à les reconnecter, il faudrait déjà que les différentes Aires naturelles protégées aient une existence au-delà du légal et des moyens pour fonctionner, ce qui est loin d'être le cas partout. 
Au-delà de ce décalage dans la mise en œuvre, on doit prendre en compte les imaginaires en jeu à ce niveau de gouvernance. Les instruments de conservation par le marché qui se succèdent depuis les années 90 - depuis les contrats de bioprospection jusqu'aux mécanismes de compensation, en passant par les REDD ou les paiements pour service environnementaux - ne réalisent bien souvent ni conservation ni marché effectif, mais on renouvelle chaque fois la promesse que l'on va pouvoir conserver la nature grâce au marché. Malgré les échecs, tant qu'on continuera à croire à cette promesse, on peut relancer un nouveau cycle. Je ne dis pas que la gouvernance globale de l'environnement n'a aucun effet, mais qu'elle permet souvent de faire autre chose que ce qu'elle prétend faire. Par exemple diffuser des cadrages dominants ou encore alimenter un marché de l'expertise. C'est tous ces multiples déphasages qu'il faut étudier et déconstruire je pense, d'où l'intérêt de combiner des approches qui font varier les niveaux d'analyse et les disciplines.

Plus récemment vous avez participé à plusieurs groupes de recherche sur l'enjeu climatique. Comment $y$ avez-vous traité la dimension transnationale?

Pour moi, le transnational, c'est ce qui se joue au-delà des États-nations, soit entre un État et une firme, soit par exemple entre ONG sur des questions dépassant les frontières nationales. Nous nous sommes plus intéressés à la fabrique de la gouvernance globale, avec ses chiffres, ses discours, ses images, qu'au transnationalisme à proprement parler, mais c'est évidemment une dimension qui compose également le global. L'existence d'un mouvement climatique transnational est ainsi un objet d'étude passionnant, notamment dans sa difficulté à exister en dehors du cadre international des négociations dont il dit généralement ne plus attendre grand-chose mais dont il a le plus grand mal à s'émanciper. Dans le projet Climacop, on s'est également intéressé aux plates-formes transnationales d'entreprises et à leur difficulté à produire un discours commun audelà des conflits entre intérêts sectoriels très différents. C'est d'ailleurs intéressant de voir comment, malgré la prétention à porter des solutions dans la lutte contre le réchauffement climatique, ce type de plates-formes reproduit les travers des institutions internationales en n'étant capable de se mettre d'accord uniquement sur un socle minimaliste autour de concepts flous comme le carbon pricing.

On a aussi analysé dans notre projet comment les fondations philanthropiques sont des acteurs très importants au niveau transnational sur les questions climatiques, notamment dans leur capacité à produire des cadrages largement repris dans les discours des institutions internationales, des entreprises et des grandes ONG. Non seulement elles ont des moyens financiers et humains considérables, mais leur position centrale à l'intersection des mondes privés, publics et de la société civile leur donne une influence inversement proportionnelle à leur discrétion. C’est mon collègue Edouard Morena (2016) qui a particulièrement mis ça en lumière. Cela dit, quand on s'intéresse à un objet comme une COP, on se rend compte que les négociations, au moins, restent avant tout une affaire internationale au sens où, au moins au niveau des négociations, ce sont les jeux et rapports de force entre États qui prédominent en dernière instance. La gouvernance globale du climat dépasse largement le jeu des négociations, mais une 
des difficultés réside précisément dans le fait qu'on essaie de gérer un problème global dans un cadre qui reste essentiellement international.

Ces projets collectifs ont notamment eu une dimension pluridisciplinaire. Considérez-vous que la pluridisciplinarité est particulièrement importante pour les enjeux environnementaux?

Oui, c'est évident. Pour ma part, j’ai toujours travaillé avec des collègues d'autres disciplines des sciences sociales: des historiens, anthropologues, économistes, politologues... Il y a au moins en France un projet de plus en plus cohérent d'humanités environnementales et ça me semble être une très bonne chose. Inversement, quand je me retrouve dans des congrès de sociologie générale, j'ai l'impression de parler un peu une autre langue et que l'environnement n'est pas encore reconnu comme un objet légitime par cette discipline. Je n'ai jamais développé de projet couplant sciences sociales et sciences de la vie par exemple et c'est sans doute dommage ; il faudrait arriver à ça même s'il ne faut pas décréter la pluridisciplinarité. En fait, plus que la dimension pluridisciplinaire, on revendique dans ces projets une dimension collaborative et conviviale avec notamment une large mise à disposition et un large partage des données et des analyses entre les membres du projet.

On a eu aussi le souci particulier de maintenir une ambiance de travail favorable et de la confiance. L'idée est assez simple, mais ce n'est pas si facile à maintenir du début du projet à la fin. En l'occurrence en amont de l'événement transnational, dans la phase de préparation, on échange un maximum d'informations sur la préparation des négociations, mais aussi sur des aspects plus matériels et logistiques (les accréditations notamment). Pendant la COP elle-même, on a des moments d'observation croisée sur certains événements et surtout des moments de débrief très réguliers pour échanger en permanence nos analyses et faire émerger des questions de recherche. Après l'événement, au moment de l'écriture, on a organisé des week-ends de discussions pour lancer le processus puis relire et commenter de manière croisée, les textes. Tout ça n'est pas spécifique aux enjeux environnementaux ni peut-être même aux événements transnationaux, mais chercher à maintenir des formes de convivialité dans n'importe quel projet me semble toujours plus agréable évidemment, mais surtout intellectuellement plus productif. Ça va un peu contre les grosses machineries institutionnelles promues au niveau national ou européen où il faut collaborer sous prétexte que l'on partage les mêmes bouts d'objets...

Quelles sont selon vous actuellement les grandes priorités de recherche sur la question des enjeux environnementaux transnationaux?

Ce n'est pas vraiment à moi de définir quelles doivent être ces priorités, mais je peux vous dire ce qu'il me semble à approfondir. Du point de vue des sciences sociales, si on arrive déjà à mieux comprendre comment se fabriquent ces enjeux et comment fonctionnent ces chaînes de traductions entre niveaux et acteurs, ça serait déjà pas mal. Les nouvelles formes de gouvernance basées sur la soft law, les signaux plutôt que la contrainte, la vérification plutôt que la sanction comme c'est le cas de l'accord de Paris qui 
en représente un cas archétypal, méritent à mon avis d'être analysées et discutées audelà du présupposé qui veut qu'elles soient plus efficaces. Quel est ce nouvel esprit de la gouvernance globale et comment performe-t-il ? Les actions du secteur privé mériteraient aussi des études de cas détaillées pour voir comment il fonctionne concrètement au-delà, ou plutôt en deçà, de visions structurelles sur le capitalisme. À un niveau plus théorique, comment se co-construisent les imaginaires et les matérialités de ces enjeux environnementaux me semblent une piste de réflexion passionnante. Ce ne sont pas les idées qui manquent donc, mais de là à dire que c'est prioritaire, je ne pense pas. Si par prioritaire, on entend des recherches qui vont dans le sens d'une amélioration de la situation par rapport à ces enjeux environnementaux, j'ai malheureusement beaucoup moins d'idées, car là, je crois que les sciences sociales et autres ont fait le travail, notamment en posant un diagnostic sans appel sur la crise environnementale avec par exemple le concept d'anthropocène. Je ne crois pas que la solution aux différentes dimensions de la crise environnementale soit scientifique, ou bien alors pour générer toujours plus de conscience collective de cette crise ; c'est un problème plus culturel ou civilisationnel qui renvoie à nos modes de production et de consommation et à notre volonté et à la possibilité même d’en changer.

\section{Bibliographie}

AYKuT S., FOYER J., MORENA E. (2017), Globalising the Climate: COP21 and the climatisation of global debates, Londres, Routledge.

Blanc G., Demeulenaere É., Feuerhahn W. (dir.), (2017), Humanités environnementales. Enquêtes et contre-enquêtes. Paris, Publications de la Sorbonne.

CARRIER, J.G. West, P., (2009), Virtualism, governance, and practice: vision and execution in environmental Conservation, New York, Berghahn Press.

FOYER J. (dir.) (2015), Regards croisés sur Rio+20, la modernisation écologique à l'épreuve, Paris, CNRS éditions.

FOYER, J., (2012) « Le réseau global des experts-militants de la biodiversité : un acteur central des controverses socio-techniques », Hermès, vol. 64, p. 155-163.

MORENA E. (2016), The price of climate action: philanthropic foundations in the international climate debate, Cham, Palgrave Macmillan.

MÜLLER, B. (2013), The gloss of harmony: the politics of policy-making in multilateral organisations, Londres, Pluto Press.

Rokström, J., StefFen, W., None, K., Personn A., Chapin F. S., F., LAmbin E., LEnTON T.M., et al. (2009), "Planetary boundaries: Exploring the safe operating space for humanity », Ecology er Society, vol. 14, n 2. 